\title{
Inductive entanglement classification of four qubits under stochastic local operations and classical communication
}

\author{
L. Lamata, ${ }^{1, *}$ J. León, ${ }^{1, \dagger}$ D. Salgado, ${ }^{2, \ddagger}$ and E. Solano ${ }^{3,4, \S}$ \\ ${ }^{1}$ Instituto de Matemáticas y Física Fundamental, CSIC, Serrano 113-bis, 28006 Madrid, Spain \\ ${ }^{2}$ Dpto. Física Teórica, Universidad Autónoma de Madrid, 28049 Cantoblanco, Madrid, Spain \\ ${ }^{3}$ Physics Department, ASC, and CeNS, Ludwig-Maximilians-Universität, Theresienstrasse 37, 80333 Munich, Germany \\ ${ }^{4}$ Sección Física, Departamento de Ciencias, Pontificia Universidad Católica del Perú, Apartado Postal 1761, Lima, Peru
}

(Received 3 November 2006; published 21 February 2007)

\begin{abstract}
Using an inductive approach to classify multipartite entangled states under stochastic local operations and classical communication introduced recently by the authors [Phys. Rev. A 74, 052336 (2006)], we give the complete classification of four-qubit entangled pure states. Apart from the expected degenerate classes, we show that there exist eight inequivalent ways to entangle four qubits. In this respect, permutation symmetry is taken into account and states with a structure differing only by parameters inside a continuous set are considered to belong to the same class.
\end{abstract}

DOI: 10.1103/PhysRevA.75.022318

PACS number(s): 03.67.Mn, 03.65.Ud, 02.10.Yn

\section{INTRODUCTION}

Entanglement is the distinguishing feature of quantum systems $[1,2]$ and is especially useful in using such systems to process information $[3,4]$. Despite its relevance this property is not yet fully understood and different questions remain open [5]. Among them a complete classification of entangled states under adequately chosen criteria of equivalence stands as a formidable task. Two of these criteria appear as outstanding: namely the so-called local unitary (LU) and stochastic local operations and classical communication (SLOCC) equivalences. The former is mathematically expressed by the relation $|\psi\rangle \sim_{L U}|\phi\rangle \Leftrightarrow|\psi\rangle=U^{[1]} \otimes \cdots$ $\otimes U^{[N]}|\phi\rangle$ for unitary matrices $U^{[k]}$, whereas the latter is mathematically translated as $|\psi\rangle \sim_{\text {SLOCC }}|\phi\rangle \Leftrightarrow|\psi\rangle=F^{[1]}$ $\otimes \cdots \otimes F^{[N]}|\phi\rangle$ for nonsingular matrices $F^{[k]}$. Although these entanglement equivalence definitions can be straightforwardly generalized to include mixed states, we will restrict ourselves to the pure state case. Furthermore, we will center upon the identification of entanglement classes under the second criterion.

The SLOCC equivalence (also known as local filtering operations) was introduced in [6] and enjoys a clear physical motivation: two multipartite pure states $|\psi\rangle$ and $|\phi\rangle$ are equivalent under SLOCC if one can be obtained from the other with non-null probability using only local operations upon each subsystem and classical communication among the different parties. In this manner, the complete classification of entanglement for $N=3$ qubits can be derived; namely, any genuinely entangled three-qubit systems is equivalent either to the Greenberger-Horne-Zeilinger (GHZ) state or to the $W$ state. The difficulties for more general cases-i.e. for $N \geq 4$-was also pointed out in Ref. [6], where a nonenumerable set of entanglement classes would be required.

\footnotetext{
*Electronic address: lamata@imaff.cfmac.csic.es

${ }^{\dagger}$ Electronic address: leon@imaff.cfmac.csic.es

*Electronic address: david.salgado@uam.es

${ }^{\S}$ Electronic address: enrique.solano@physik.lmu.de
}

In this work, using our preceding inductive approach [7], we give the complete classification of entanglement for $N$ $=4$ qubits. Although this was already apparently performed in Ref. [8], we argue that their enumeration does not follow the same philosophy as in the seminal paper by Dür et al. $[6,9]$. In Sec. II, we recopile the preceding relevant results which will be later used in Sec. III to obtain in detail all entanglement classes under SLOCC. Our concluding remarks are presented in Sec. IV.

\section{REVIEW OF THE INDUCTIVE METHOD}

The forthcoming entanglement classification for $N$ qubits is based on the inductive approach which the present authors introduced in Ref. [7] and in some auxiliary results which were proved therein. The main idea of this approach was to establish a classification of the right singular subspace of the coefficient matrix of each pure state $|\psi\rangle\rangle$ expressed in an adequate product basis (typically the canonicalcomputational-basis). This classification must be carried out according to the entanglement classes for $N-1$ qubits (hence the term inductive). Consequently, knowing in advance that there are only two entanglement classes for two qubits - namely, the product class 00 and the entanglement class $\Psi$-the right singular subspace $\mathfrak{W}$ of the coefficient matrix of the state of any three-qubit system results to be of six possible types: i.e. two one-dimensional subspaces $\mathfrak{W}=\operatorname{span}\{|\phi \psi\rangle\}$ and $\mathfrak{W}=\operatorname{span}\{|\Psi\rangle\}$ driving us to the degenerate classes 000 and $0_{1} \Psi_{23}$, respectively; two twodimensional product subspaces $\mathfrak{W}=|\phi\rangle \otimes \mathrm{C}^{2}$ and $\mathfrak{W}=\mathrm{C}^{2}$ $\otimes|\psi\rangle$, driving us to the degenerate classes $0_{2} \Psi_{13}$ and $0_{3} \Psi_{12}$, respectively; and, finally, another two two-dimensional classes $\mathfrak{W}=\operatorname{span}\{00,00\}$ and $\mathfrak{W}=\operatorname{span}\{00, \Psi\}$, driving us to the well-known GHZ and $W$ genuine entanglement classes. Let us recall that $\mathfrak{W}=\operatorname{span}\{00, \Psi\}$ stands for the fact that the subspace $\mathfrak{W}$ contains (up to normalization) only one product vector (i.e., only one of type 00 ). This will sometimes be also expressed as $\mathfrak{W}=\operatorname{span}\{|\phi \varphi\rangle,|\Psi\rangle\}$. This convention will be profusely followed in the next section. We will employ these 
six tripartite entanglement classes to find those of $N=4 \mathrm{qu}-$ bits.

Moreover, in the proof, we will make use of the following results, already proved in [7].

Proposition 1. Let $\mathfrak{W}$ be a two-dimensional subspace in $\mathrm{C}^{2} \otimes \mathbb{C}^{2}$. Then $\mathfrak{W}=\operatorname{span}\{|\phi \varphi\rangle,|\Psi\rangle\} \quad$ if, and only if, $\mathfrak{W}=\operatorname{span}\{|\phi \varphi\rangle,|\phi \bar{\varphi}\rangle+|\bar{\phi} \varphi\rangle\}$, where the overbar denotes linear independence.

Let us also recall that only two options are left for the structure of any two-dimensional subspace [10] - namely, $\mathfrak{W}=\operatorname{span}\{00,00\}$ and $\mathfrak{W}=\operatorname{span}\{00, \Psi\}$, where the above convention has been used. Complementarily, the following criterion to discern whether a given tripartite pure state belongs to any of the six entanglement classes will be also necessary. We previously needed the following definition.

Definition 1. Let

$$
\begin{gathered}
C^{(1)} \equiv C_{1 \mid 23}=\left(\begin{array}{llll}
c_{111} & c_{112} & c_{121} & c_{122} \\
c_{211} & c_{212} & c_{221} & c_{222}
\end{array}\right), \\
C^{(2)} \equiv C_{2 \mid 13}=\left(\begin{array}{llll}
c_{111} & c_{112} & c_{211} & c_{212} \\
c_{121} & c_{122} & c_{221} & c_{222}
\end{array}\right),
\end{gathered}
$$

and

$$
C^{(3)} \equiv C_{3 \mid 12}=\left(\begin{array}{llll}
c_{111} & c_{121} & c_{211} & c_{221} \\
c_{112} & c_{122} & c_{212} & c_{222}
\end{array}\right)
$$

be the coefficient matrices of the partitions $1|23,2| 13$, and $3 \mid 12$, respectively, of the tripartite pure state $|\Psi\rangle=\sum_{i j k} c_{i j k}|i-1\rangle \otimes|j-1\rangle \otimes|k-1\rangle$. We will define the matrices $W_{1}$ and $W_{2}$ as

$$
W_{1}=\left(\begin{array}{ll}
c_{111} & c_{112} \\
c_{121} & c_{122}
\end{array}\right), \quad W_{2}=\left(\begin{array}{ll}
c_{211} & c_{212} \\
c_{221} & c_{222}
\end{array}\right) .
$$

With these definitions and remembering that the spectrum of a matrix $A$ is denoted as $\sigma(A)$, in [7] we proved the following.

Theorem 1. Let $|\Psi\rangle$ denote the pure state of a tripartite system and $C^{(i)}$ its coefficient matrix according to the partition $i \mid j k$. Then,

(1) $|\Psi\rangle$ belongs to the 000 class if, and only if, $r\left(C^{(i)}\right)=1$ for all $i=1,2,3$.

(2) $|\Psi\rangle$ belongs to the $0_{1} \Psi_{23}$ class if, and only if, $r\left(C^{(1)}\right)=1$ and $r\left(C^{(k)}\right)=2$ for $k=2,3$.

(3) $|\Psi\rangle$ belongs to the $0_{2} \Psi_{13}$ class if, and only if, $r\left(C^{(2)}\right)=1$ and $r\left(C^{(k)}\right)=2$ for $k=1,3$.

(4) $|\Psi\rangle$ belongs to the $0_{3} \Psi_{12}$ class if, and only if, $r\left(C^{(3)}\right)=1$ and $r\left(C^{(k)}\right)=2$ for $k=1,2$.

(5) $|\Psi\rangle$ belongs to the GHZ class if, and only if, one of the following situations occurs:

(i) $r\left(C^{(i)}\right)=2$ for all $i=1,2,3$ and $r\left(W_{1}\right)=r\left(W_{2}\right)=1$.

(ii) $r\left(C^{(i)}\right)=2$ for all $i=1,2,3, r\left(W_{j}\right)=2, r\left(W_{k}\right)=1$, and $\sigma\left(W_{j}^{-1} W_{k}\right)$ is nondegenerate.

(iii) $r\left(C^{(i)}\right)=2$ for all $i=1,2,3, r\left(W_{1}\right)=2, r\left(W_{2}\right)=2$, and $\sigma\left(W_{1}^{-1} W_{2}\right)$ is nondegenerate.
(6) $|\Psi\rangle$ belongs to the $W$ class if, and only if, one of the following situations occurs:

(i) $r\left(C^{(i)}\right)=2$ for all $i=1,2,3, r\left(W_{j}\right)=2, r\left(W_{k}\right)=1$, and $\sigma\left(W_{j}^{-1} W_{k}\right)$ is degenerate.

(ii) $r\left(C^{(i)}\right)=2$ for all $i=1,2,3, r\left(W_{1}\right)=2, r\left(W_{2}\right)=2$, and $\sigma\left(W_{1}^{-1} W_{2}\right)$ is degenerate.

\section{FOUR-QUBIT CLASSIFICATION}

The classification inductive procedure for $N=4$ qubits is long, although systematic. We will first detect the degenerate classes, which furthermore are predictable in advance: 0000, $0_{i_{1}} 0_{i_{2}} \Psi_{i_{3} i_{4}}, 0_{i_{1}}$ GHZ, $0_{i_{1}} W$, and $\Psi_{i_{1} i_{2}} \Psi_{i_{3} i_{4}}$, with $i_{k}=1,2,3,4$. Then, we will work out the genuine classes. The classification is performed according to the structure of the right singular subspace of the coefficient matrix of the four-partite pure states: $\mathfrak{W}=\operatorname{span}\left\{\Psi_{i}, \Psi_{j}\right\}$, where $\Psi_{i, j}=000,0_{k} \Psi, \mathrm{GHZ}, W$.

\section{A. Degenerate classes}

As stated we will revise in turns each of the possible structures of the right singular subspace $\mathfrak{W}$ of the coefficient matrix. Remember that now $\mathfrak{W} \subset \mathbb{C}^{2} \otimes \mathbb{C}^{2} \otimes \mathbb{C}^{2}$.

(1) $\mathfrak{W}=\operatorname{span}\{000\}$. In this case, we can write $\mathfrak{W}=\operatorname{span}\{|\phi \varphi \psi\rangle\}$. Mimicking the same arguments as in [7] (see also below for the genuine classes), we obtain the canonical vector $|0000\rangle$, corresponding to the degenerate class 0000 .

(2) $\mathfrak{W}=\operatorname{span}\left\{0_{1} \Psi_{23}\right\}$. This drives us to the canonical vector $|0000\rangle+|0011\rangle$, corresponding to the degenerate class $0_{1} 0_{2} \Psi_{34}$.

(3) $\mathfrak{W}=\operatorname{span}\left\{0_{2} \Psi_{13}\right\}$. This drives us to the canonical vector $|0000\rangle+|0101\rangle$, corresponding to the degenerate class $0_{1} 0_{3} \Psi_{24}$.

(4) $\mathfrak{W}=\operatorname{span}\left\{0_{3} \Psi_{12}\right\}$. This drives us to the canonical vector $|0000\rangle+|0110\rangle$, corresponding to the degenerate class $0_{1} 0_{4} \Psi_{23}$.

(5) $\mathfrak{W}=\operatorname{span}\{\mathrm{GHZ}\}$. This drives us to the canonical vector $|0000\rangle+|0111\rangle$, corresponding to the degenerate class $0_{1} \mathrm{GHZ}$.

(6) $\mathfrak{W}=\operatorname{span}\{W\}$. This drives us to the canonical vector $|0001\rangle+|0010\rangle+|0100\rangle$, corresponding to the degenerate class $0_{1} W$.

(7) $\mathfrak{W}=\operatorname{span}\{000,000\}$. When $\mathfrak{W}=|\phi \varphi\rangle \otimes \mathbb{C}^{2}$, we obtain the canonical vector $|0000\rangle+|1001\rangle$, corresponding to the degenerate class $\mathrm{O}_{2} \mathrm{O}_{3} \Psi_{14}$.

(8) $\mathfrak{W}=\operatorname{span}\{000,000\}$. When $\mathfrak{W}=|\phi\rangle \otimes \mathbb{C}^{2} \otimes|\psi\rangle$, we obtain the canonical vector $|0000\rangle+|1010\rangle$, corresponding to the degenerate class $0_{2} \mathrm{O}_{4} \Psi_{13}$.

(9) $\mathfrak{W}=\operatorname{span}\{000,000\}$. When $\mathfrak{W}=\mathbb{C}^{2} \otimes|\varphi \psi\rangle$, we obtain the canonical vector $|0000\rangle+|1100\rangle$, corresponding to the degenerate class $0_{3} 0_{4} \Psi_{12}$.

(10) $\mathfrak{W}=\operatorname{span}\{000,000\}$. When $\mathfrak{W}=|\phi\rangle$ $\otimes \operatorname{span}\{|\varphi \psi\rangle,|\bar{\varphi} \bar{\psi}\rangle\}$, we obtain the canonical vector $|0000\rangle$ $+|1011\rangle$, corresponding to the degenerate class $0_{2} \mathrm{GHZ}$. 
(11) $\mathfrak{W}=\operatorname{span}\{000,000\}$. When $\mathfrak{W}=\operatorname{span}\{|\phi \varphi \psi\rangle,|\bar{\phi} \varphi \bar{\psi}\rangle\}$, we obtain the canonical vector $|0000\rangle+|1101\rangle$, corresponding to the degenerate class $\mathrm{O}_{3} \mathrm{GHZ}$.

(12) $\mathfrak{W}=\operatorname{span}\{000,000\}$. When $\mathfrak{W}=\operatorname{span}\{|\phi \varphi \psi\rangle,|\bar{\phi} \bar{\varphi} \psi\rangle\}$, we obtain the canonical vector $|0000\rangle+|1110\rangle$, corresponding to the degenerate class $0_{4} \mathrm{GHZ}$.

(13) $\mathfrak{W}=\operatorname{span}\left\{000,0_{1} \Psi_{23}\right\}$. When $\mathfrak{W}=|\phi\rangle$ $\otimes \operatorname{span}\left\{\left|\varphi_{1} \psi_{1}\right\rangle,\left|\bar{\varphi}_{2} \bar{\psi}_{2}\right\rangle\right\}$, we obtain the canonical vector $|0001\rangle+|0010\rangle+|1000\rangle$, corresponding to the degenerate class $0_{2} W$.

(14) $\mathfrak{W}=\operatorname{span}\left\{000,0_{2} \Psi_{13}\right\} . \quad$ When $\mathfrak{W}$ $=\operatorname{span}\left\{\left|\phi_{1} \varphi \psi_{1}\right\rangle,\left|\phi_{2} \varphi \psi_{2}\right\rangle+\left|\bar{\phi}_{2} \varphi \bar{\psi}_{2}\right\rangle\right\}$, we obtain the canonical vector $|0001\rangle+|0100\rangle+|1000\rangle$, corresponding to the degenerate class $\mathrm{O}_{3} \mathrm{~W}$.

(15) $\mathfrak{W}=\operatorname{span}\left\{000,0_{3} \Psi_{12}\right\}$. When $\mathfrak{W}$ $=\operatorname{span}\left\{\left|\phi_{1} \varphi_{1}\right\rangle,\left|\phi_{2} \varphi_{2}\right\rangle+\left|\bar{\phi}_{2} \bar{\varphi}_{2}\right\rangle\right\} \otimes|\psi\rangle$, we obtain the canonical vector $|0010\rangle+|0100\rangle+|1000\rangle$, corresponding to the degenerate class $0_{4} W$.

(16) $\mathfrak{W}=\operatorname{span}\left\{0_{1} \Psi_{13}, 0_{1} \Psi_{13}\right\}$. When $\mathfrak{W}=\mathbb{C}^{2} \otimes \operatorname{span}\{|\Psi\rangle\}$, we obtain the canonical vector $|0000\rangle+|0011\rangle+|1100\rangle$ $+|1111\rangle=(|00\rangle+|11\rangle)(|00\rangle+|11\rangle)$, corresponding to the degenerate class $\Psi_{12} \Psi_{34}$.

(17) $\mathfrak{W}=\operatorname{span}\left\{0_{2} \Psi_{13}, 0_{2} \Psi_{13}\right\}$. When $\mathfrak{W}=\operatorname{span}\{|\phi \varphi \psi\rangle$ $+|\bar{\phi} \varphi \bar{\psi}\rangle,|\phi \bar{\varphi} \psi\rangle+|\bar{\phi} \bar{\varphi} \bar{\psi}\rangle\}$, we obtain the canonical vector $|0000\rangle+|0101\rangle+|1010\rangle+|1111\rangle=(|00\rangle+|11\rangle)_{13}(|00\rangle+|11\rangle)_{24}$, corresponding to the degenerate class $\Psi_{13} \Psi_{24}$.

(18) $\mathfrak{W}=\operatorname{span}\left\{0_{3} \Psi_{13}, 0_{3} \Psi_{13}\right\}$. When $\mathfrak{W}=|\Psi\rangle \otimes \mathrm{C}^{2}$, we obtain the canonical vector $|0000\rangle+|0110\rangle+|1001\rangle+|1111\rangle$ $=(|00\rangle+|11\rangle)_{14}(|00\rangle+|11\rangle)_{23}$, corresponding to the degenerate class $\Psi_{14} \Psi_{23}$.

No further degenerate classes can be found within the rest of possible structures for $\mathfrak{W}$. Notice how all of them can be obtained from the first 6 and 16th cases by just permuting the qubit indices. We have preferred to make use of a systematic approach in order to illustrate its usage.

\section{B. Genuine classes}

In order to be exhaustive we must check out all possible structures that the right singular subspace $\mathfrak{W}$ may have. An $a$ priori list is given by $\operatorname{span}\{000,000\}, \operatorname{span}\left\{000,0_{i_{1}} \Psi\right\}$, $\operatorname{span}\{000, \mathrm{GHZ}\}, \quad \operatorname{span}\{000, W\}, \quad \operatorname{span}\left\{0_{i_{1}} \Psi, 0_{i_{2}} \Psi\right\}$, $\operatorname{span}\left\{0_{i_{1}} \Psi, \mathrm{GHZ}\right\}, \quad \operatorname{span}\left\{0_{i_{1}} \Psi, W\right\}, \quad \operatorname{span}\{\mathrm{GHZ}, \mathrm{GHZ}\}$, $\operatorname{span}\{\mathrm{GHZ}, W\}$, and $\operatorname{span}\{W, W\}$, where $i_{k}=1,2,3$. The same convention as above has been followed when we say that, e.g., $\operatorname{span}\left\{0_{i_{1}} \Psi, \mathrm{GHZ}\right\}$ indicates that only one vector belonging to the class $0_{i_{1}} \Psi$ is contained in $\mathfrak{W}$, the rest being all GHZ (and possibly $W$ ). In other words, in $\operatorname{span}\left\{\Psi_{i}, \Psi_{k}\right\}$ it is understood that $\Psi_{i}$ and $\Psi_{k}$ are those vectors in $\mathfrak{W}$ with the lowest degree of entanglement degeneracy, according to the scale $000<0_{i_{k}} \Psi<\mathrm{GHZ}, W$.

\section{1. $\mathfrak{W}=\operatorname{span}\{000,000\}$}

All cases leading to degenerate cases have been considered above. However, when $\mathfrak{W}=\operatorname{span}\{|\phi \varphi \psi, \bar{\phi} \bar{\varphi} \bar{\psi}\rangle\}$, a wellknown entanglement class arises: namely, the GHZ class. We choose nonsingular transformations $F^{[2]}$ so that $|0\rangle=F^{[2]}|\phi\rangle$, $|1\rangle=F^{[2]}|\bar{\phi}\rangle$ and similarly for $F^{[3,4]}$. We choose also $F^{[1]}$ so that $\left[F^{[1]}\left|\mathbf{v}_{1}\right\rangle F^{[1]}\left|\mathbf{v}_{2}\right\rangle\right]=\left[\mu_{i j}^{*}\right]^{-1}\left(\begin{array}{cc}\sigma_{1}^{-1} & 0 \\ 0 & \sigma_{2}^{-1}\end{array}\right)$, where $\left|\mathbf{v}_{j}\right\rangle$ denotes the left singular vectors of the coefficient matrix $C_{\Phi}$ of the state $|\Phi\rangle \in \mathrm{C}_{1}^{2} \otimes \mathrm{C}_{1}^{2} \otimes \mathrm{C}_{1}^{2} \otimes \mathrm{C}^{2}$ and $\mu_{i j}$ are the coefficients expressing the right singular vectors $\left|\mathbf{w}_{k}\right\rangle$ of $C_{\Phi}$ in terms of $|\phi \varphi \psi\rangle$ and $|\bar{\phi} \bar{\varphi} \bar{\psi}\rangle:\left|\mathbf{w}_{i}\right\rangle=\mu_{i 1}|\phi \varphi \psi\rangle+\mu_{i 2}|\bar{\phi} \bar{\varphi} \bar{\psi}\rangle$. Then, we can write [7]

$$
\begin{aligned}
C_{F^{[1]]} \cdots \otimes F^{[4]} \mid \Phi}= & \left(\mu_{i j}^{*}\right)^{-1}\left(\begin{array}{cc}
\sigma_{1} & 0 \\
0 & \sigma_{2}
\end{array}\right)^{-1}\left(\begin{array}{ccccc}
\sigma_{1} & 0 \\
0 & \sigma_{2}
\end{array}\right) \\
& \times\left(\begin{array}{ccccccccc}
\mu_{11}^{*} & 0 & 0 & 0 & 0 & 0 & 0 & \mu_{12}^{*} \\
\mu_{21}^{*} & 0 & 0 & 0 & 0 & 0 & 0 & \mu_{22}^{*}
\end{array}\right) \\
& =\left(\begin{array}{cccccccc}
1 & 0 & 0 & 0 & 0 & 0 & 0 & 0 \\
0 & 0 & 0 & 0 & 0 & 0 & 0 & 1
\end{array}\right) .
\end{aligned}
$$

This matrix corresponds to the canonical state $|0000\rangle$ $+|1111\rangle$-i.e., to the so-called GHZ state.

\section{2. $\mathfrak{W}=\operatorname{span}\left\{000,0_{1} \Psi_{23}\right\}$}

In general, in this case $\mathfrak{W}=\operatorname{span}\left\{\left|\phi_{1} \varphi_{1} \psi_{1}\right\rangle,\left|\phi_{2}\right\rangle \otimes\left(\left|\varphi_{2} \psi_{2}\right\rangle\right.\right.$ $\left.\left.+\left|\bar{\varphi}_{2} \bar{\psi}_{2}\right\rangle\right)\right\}$. The degenerate case takes place when $\left|\phi_{1}\right\rangle$ and $\left|\phi_{2}\right\rangle$ are linearly dependent, driving us to the class $0_{2} W$, already stated above.

Thus, let us consider the case when $\left\{\left|\phi_{1}\right\rangle,\left|\phi_{2}\right\rangle\right\}$ are linearly independent. Now the two-dimensional subspace $\operatorname{span}\left\{\left|\varphi_{1} \psi_{1}\right\rangle,\left|\varphi_{2} \psi_{2}\right\rangle+\left|\bar{\varphi}_{2} \bar{\psi}_{2}\right\rangle\right\} \quad$ can be either of type $\operatorname{span}\{00,00\}$ or of type $\operatorname{span}\{00, \Psi\}$ :

(i) When $\operatorname{span}\left\{\left|\varphi_{1} \psi_{1}\right\rangle,\left|\varphi_{2} \psi_{2}\right\rangle+\left|\bar{\varphi}_{2} \bar{\psi}_{2}\right\rangle\right\}=\operatorname{span}\{00,00\}$, then there exist linearly independent vectors $|\phi\rangle,|\bar{\phi}\rangle,|\varphi \psi\rangle$, and $|\bar{\varphi} \bar{\psi}\rangle \quad$ such that $\quad\left|\mathbf{w}_{k}\right\rangle=\mu_{k 1}|\phi \varphi \psi\rangle+\mu_{k 2}|\bar{\phi}\rangle \otimes(|\varphi \psi\rangle$ $+|\bar{\varphi} \bar{\psi}\rangle)$. Thus, it is immediate to realize that there exist nonsingular transformations $F^{[k]}, k=1,2,3,4$, such that

$$
\begin{aligned}
C_{F^{[1]}{ }_{\otimes \ldots \otimes F^{[4]} \mid \Phi}=} & \left(\mu_{i j}^{*}\right)^{-1}\left(\begin{array}{cc}
\sigma_{1} & 0 \\
0 & \sigma_{2}
\end{array}\right)^{-1}\left(\begin{array}{cccc}
\sigma_{1} & 0 \\
0 & \sigma_{2}
\end{array}\right) \\
& \times\left(\begin{array}{cccccccc}
\mu_{11}^{*} & 0 & 0 & 0 & \mu_{12}^{*} & 0 & 0 & \mu_{12}^{*} \\
\mu_{21}^{*} & 0 & 0 & 0 & \mu_{22}^{*} & 0 & 0 & \mu_{22}^{*}
\end{array}\right) \\
= & \left(\begin{array}{cccccccc}
1 & 0 & 0 & 0 & 0 & 0 & 0 & 0 \\
0 & 0 & 0 & 0 & 1 & 0 & 0 & 1
\end{array}\right) .
\end{aligned}
$$

This canonical coefficient matrix corresponds to the state $|0000\rangle+|1100\rangle+|1111\rangle$.

(ii) When $\operatorname{span}\left\{\left|\varphi_{1} \psi_{1}\right\rangle,\left|\varphi_{2} \psi_{2}\right\rangle+\left|\bar{\varphi}_{2} \bar{\psi}_{2}\right\rangle\right\}=\operatorname{span}\{00, \Psi\}$, then there exist vectors $|\phi\rangle,|\bar{\phi}\rangle,|\varphi\rangle,|\bar{\varphi}\rangle,|\psi\rangle$, and $|\bar{\psi}\rangle$ such that $\left|\mathbf{w}_{k}\right\rangle=\mu_{k 1}|\phi \varphi \psi\rangle+\mu_{k 2}|\bar{\phi}\rangle \otimes(|\varphi \bar{\psi}\rangle+|\bar{\varphi} \psi\rangle)$. Thus, it is immediate to realize that there exist nonsingular transformations $F^{[k]}, k=1,2,3,4$, such that 


$$
\begin{aligned}
C_{\left.F^{[1]}\right]_{\left.\otimes \ldots F^{[4]}\right]} \mid \Phi}= & \left(\mu_{i j}^{*}\right)^{-1}\left(\begin{array}{cc}
\sigma_{1} & 0 \\
0 & \sigma_{2}
\end{array}\right)^{-1}\left(\begin{array}{cccc}
\sigma_{1} & 0 \\
0 & \sigma_{2}
\end{array}\right) \\
& \times\left(\begin{array}{cccccccc}
\mu_{11}^{*} & 0 & 0 & 0 & 0 & \mu_{12}^{*} & \mu_{12}^{*} & 0 \\
\mu_{21}^{*} & 0 & 0 & 0 & 0 & \mu_{22}^{*} & \mu_{22}^{*} & 0
\end{array}\right) \\
& =\left(\begin{array}{cccccccc}
1 & 0 & 0 & 0 & 0 & 0 & 0 & 0 \\
0 & 0 & 0 & 0 & 0 & 1 & 1 & 0
\end{array}\right) .
\end{aligned}
$$

This canonical coefficient matrix corresponds to the state $|0000\rangle+|1101\rangle+|1110\rangle$.

\section{3. $\mathfrak{W}=\operatorname{span}\left\{000,0_{2} \Psi_{13}\right\}$}

Although it is always possible to reproduce a similar argument to the one above, we will resort to permutation symmetry, thus leading to the degenerate class $0_{3} \mathrm{~W}$ (already stated) and the genuine classes with canonical states given by $|0000\rangle+|1010\rangle+|1111\rangle$ and $|0000\rangle+|1011\rangle+|1110\rangle$.

\section{4. $\mathfrak{W}=\operatorname{span}\left\{000,0_{3} \Psi_{12}\right\}$}

With analogous arguments, in this case the degenerate class is $0_{4} W$ (already stated) and the genuine classes are identified by the canonical states given by $|0000\rangle+|1001\rangle$ $+|1111\rangle$ and $|0000\rangle+|1011\rangle+|1101\rangle$.

\section{5. $\mathfrak{W}=\operatorname{span}\{000, G H Z\}$}

This class is highly richer than those above. In this case $\mathfrak{W}=\operatorname{span}\left\{\left|\phi_{1} \varphi_{1} \psi_{1}\right\rangle,\left|\phi_{2} \varphi_{2} \psi_{2}\right\rangle+\left|\bar{\phi}_{2} \bar{\varphi}_{2} \bar{\psi}_{2}\right\rangle\right\}$, with the restriction that no product state other than $\left|\phi_{1} \varphi_{1} \psi_{1}\right\rangle$ and no $0_{k} \Psi$ state belong to $\mathfrak{W}$ (otherwise we would be in one of the preceding classes). We define now nonsingular transformations $F^{[k]}$ such that $F^{[2]}\left|\phi_{2}\right\rangle=|0\rangle, F^{[2]}\left|\bar{\phi}_{2}\right\rangle=|1\rangle$, and similarly for $\left|\varphi_{2}\right\rangle$, $\left|\bar{\varphi}_{2}\right\rangle, \quad\left|\psi_{2}\right\rangle$, and $\left|\bar{\psi}_{2}\right\rangle$. We define also $F^{[1]}$ such that $\left[F^{[1]}\left|\mathbf{v}_{1}\right\rangle F^{[1]}\left|\mathbf{v}_{2}\right\rangle\right]=\left[\mu_{i j}^{*}\right]^{-1}\left(\begin{array}{cc}\sigma_{1}^{-1} & 0 \\ 0 & \sigma_{2}^{-1}\end{array}\right)$, with the same conventions as above. Then, the canonical vector can be written as [11]

$$
|0 \phi \varphi \psi\rangle+|1000\rangle+|1111\rangle .
$$

The factor vectors $|\phi\rangle,|\varphi\rangle$, and $|\psi\rangle$ are arbitrary up to the restriction of not producing more than one 000 vector and no $0_{k} \Psi$ vector in $\mathfrak{W}$. Although this recipe embraces all possible cases, we will single out two different subsets within this class: namely, (i) that of states leading to right singular subspaces $\mathfrak{W}$ with no $W$ state in it-i.e., containing only one product vector and GHZ vectors-and (ii) that of states leading to right singular subspaces $\mathfrak{W}$ with at least one $W$ vector in it. Indeed, we will show that there will be either one or two $W$ vectors in $\mathfrak{W}$.

(i) The set of states leading to $\mathfrak{W}$ containing no $W$ vectors is that identified by the canonical ones given by $|001 \psi\rangle+|1000\rangle+|1111\rangle,|00 \varphi 1\rangle+|1000\rangle+|1111\rangle$ and $|0 \phi 01\rangle$ $+|1000\rangle+|1111\rangle$, with no restrictions upon the factor vectors. Moreover, if we denote the components of $|\phi\rangle$ in the (nonnecessarily orthonormal) basis $\left\{\left|\phi_{2}\right\rangle,\left|\bar{\phi}_{2}\right\rangle\right\}$ by $\phi_{0}$ and $\phi_{1}$ and similarly for $|\varphi\rangle$ and $|\psi\rangle$, then whenever $0 \neq \sqrt{\phi_{0} \varphi_{0} \psi_{0}}$ $= \pm \sqrt{\phi_{1} \varphi_{1} \psi_{1}} \neq 0$, there will also be a unique product vector, the rest being all GHZ in the right singular vector of the generic state (6).

This is proved resorting to theorem 1 . We will illustrate the calculation with the first canonical vector $|001 \psi\rangle$ $+|1000\rangle+|1111\rangle$, whose right singular subspace is spanned by $|01 \psi\rangle$ and $\frac{1}{\sqrt{2}}|000\rangle+\frac{1}{\sqrt{2}}|111\rangle$. Thus, a generic vector in $\mathfrak{W}$ will be of the form $\alpha(|000\rangle+|111\rangle)+\beta|01 \psi\rangle$, whose coefficient matrix is [cf. Eqs. (1a)-(1c)]

$$
C^{(1)}=\left(\begin{array}{cccc}
\alpha & 0 & \beta \psi_{0}^{*} & \beta \psi_{1}^{*} \\
0 & 0 & 0 & \alpha
\end{array}\right) .
$$

For any values of $\psi_{k}^{*}$ it is clear that $r\left(C^{(i)}\right)=2$ for $i=1,2,3$ (except for the trivial case $\alpha=0$ ). Then, if $\psi_{1}=0, r\left(W_{1}\right)$ $=r\left(W_{2}\right)=1$; thus, it is a GHZ vector; if $\psi_{1} \neq 0, r\left(W_{1}\right)=2$ and $r\left(W_{2}\right)=1$ and the spectrum of $W_{1}^{-1} W_{2}$ is never degenerate. It is again a GHZ vector for any value of $|\psi\rangle$.

The other canonical vectors are handled in a similar fashion, and they can additionally be obtained under permutations among qubits 2,3 , and 4 . Among them we single out the particular cases $|0000\rangle+|1101\rangle+|0111\rangle,|0000\rangle+|1011\rangle$ $+|0111\rangle$ and $|0000\rangle+|1110\rangle+|0111\rangle$, which can also be obtained from those in the $0_{k} \Psi$ classes by permutations among the four qubits.

For the generic state (6), let us write, as explicitly supposed, $\mathfrak{W}=\operatorname{span}\{|\phi \varphi \psi\rangle,|000\rangle+|111\rangle\}$, with no vector $|\phi\rangle$, $|\varphi\rangle$, and $|\psi\rangle$ equal to $|0\rangle$ or $|1\rangle$. If we denote again by subindices 0 and 1 the components of each vector $|\phi\rangle,|\varphi\rangle$, and $|\psi\rangle$ in the $\{|0\rangle,|1\rangle\}$ basis, then the coefficient matrix of a generic vector in $\mathfrak{W}$ will be

$$
C=\left(\begin{array}{cccc}
\alpha \phi_{0} \varphi_{0} \psi_{0}+\beta & \alpha \phi_{0} \varphi_{0} \psi_{1} & \alpha \phi_{0} \varphi_{1} \psi_{0} & \alpha \phi_{0} \varphi_{1} \psi_{1} \\
\alpha \phi_{1} \varphi_{0} \psi_{0} & \alpha \phi_{1} \varphi_{0} \psi_{1} & \alpha \phi_{1} \varphi_{1} \psi_{0} & \alpha \phi_{1} \varphi_{1} \psi_{1}+\beta
\end{array}\right),
$$

where no coefficient is null, as supposed. Then it should be clear that $r\left(C^{(i)}\right)=2$ for all $i=1,2,3$. Furthermore, since $\operatorname{det} W_{1}=\alpha \beta \phi_{0} \varphi_{1} \psi_{1}$ and $\operatorname{det} W_{2}=\alpha \beta \phi_{1} \varphi_{0} \psi_{0}$, we will have $r\left(W_{1}\right)=r\left(W_{2}\right)=2$ (except for the trivial cases $\alpha \beta=0$ ); thus, we must check the degeneracy of the spectrum of $W_{1}^{-1} W_{2}$ : $\sigma\left(W_{1}^{-1} W_{2}\right)$ is degenerate whenever the discriminant of the characteristic polynomial is null-i.e., whenever $\left[\operatorname{tr}\left(W_{1}^{-1} W_{2}\right)\right]^{2}-4 \operatorname{det}\left(W_{1}^{-1} W_{2}\right)=0$, which drives us to the condition $\beta+\alpha\left(\sqrt{\phi_{0} \varphi_{0} \psi_{0}} \pm \sqrt{\phi_{1} \varphi_{1} \psi_{1}}\right)^{2}=0$, which immediately yields the above stated conditions.

(ii) The set of states leading to $\mathfrak{W}$ containing at least one $W$ vector comprises the rest of possibilites. To prove this assertion we need to show that all possibilities for $\mathfrak{W}$ having no $W$ vector has been already considered. The most generic case occurs when $\mathfrak{W}=\operatorname{span}\left\{\left|\phi_{1} \varphi_{1} \psi_{1}\right\rangle,\left|\phi_{2} \varphi_{2} \psi_{2}\right\rangle+\left|\bar{\phi}_{2} \bar{\varphi}_{2} \bar{\psi}_{2}\right\rangle\right\}$, which we detach in the following particular cases.

(a) If $\left|\phi_{1} \varphi_{1} \psi_{1}\right\rangle=\left|\phi_{2} \varphi_{2} \psi_{2}\right\rangle,\left|\bar{\phi}_{2} \bar{\varphi}_{2} \bar{\psi}_{2}\right\rangle$, then two 000 vectors belong to $\mathfrak{W}$. This case is ruled out.

(b) If $\left|\phi_{1} \varphi_{1} \psi_{1}\right\rangle=\left|\phi_{2} \varphi_{2} \psi\right\rangle,\left|\bar{\phi}_{2} \bar{\varphi}_{2} \psi\right\rangle$, with $|\psi\rangle \neq\left|\psi_{2}\right\rangle,\left|\bar{\psi}_{2}\right\rangle$, respectively, then there will be a $0_{k} \Psi$ in $\mathfrak{W}$ unless $|\psi\rangle$ $=\left|\bar{\psi}_{2}\right\rangle,\left|\psi_{2}\right\rangle$, respectively. We give the proof for the first case. Let $\left|\phi_{1} \varphi_{1} \psi_{1}\right\rangle=\left|\phi_{2} \varphi_{2} \psi\right\rangle$, with $|\psi\rangle \neq\left|\psi_{2}\right\rangle$. A generic vector in $\mathfrak{W}$ will be of the form $\alpha\left|\phi_{2} \varphi_{2} \psi\right\rangle+\beta\left(\left|\phi_{2} \varphi_{2} \psi_{2}\right\rangle+\left|\bar{\phi}_{2} \bar{\varphi}_{2} \bar{\psi}_{2}\right\rangle\right)$ 
$=\left|\phi_{2} \varphi_{2}\right\rangle\left(\alpha|\psi\rangle+\beta\left|\psi_{2}\right\rangle\right)+\beta\left|\bar{\phi}_{2} \bar{\varphi}_{2} \bar{\psi}_{2}\right\rangle$. It is clear that, provided that $|\psi\rangle$ and $\left|\bar{\psi}_{2}\right\rangle$ are linearly independent, it is always possible to find $\alpha, \beta$ such that $\alpha|\psi\rangle+\beta\left|\bar{\psi}_{2}\right\rangle=\bar{\beta}\left|\bar{\psi}_{2}\right\rangle$, driving us to a $0_{3} \Psi$ vector in $\mathfrak{W}$, against the general hypothesis. By symmetry, all other cases can be accounted for in a similar fashion, leaving us with the cases that whenever two vectors in $|\phi \varphi \psi\rangle$ are equal to two vectors in $\left|\phi_{2} \varphi_{2} \psi_{2}\right\rangle$ or $\left|\bar{\phi}_{2} \bar{\varphi}_{2} \bar{\psi}_{2}\right\rangle$, then the other must be the third one corresponding to the opposite. For instance, if $\left|\phi_{2} \varphi_{2} \psi_{2}\right\rangle=|000\rangle,\left|\bar{\phi}_{2} \bar{\varphi}_{2} \bar{\psi}_{2}\right\rangle=|111\rangle$, and $\left|\phi_{1} \varphi_{1} \psi_{1}\right\rangle=|00 \psi\rangle$, it must be $|\psi\rangle=|1\rangle$.

(c) If $\left|\phi_{1} \varphi_{1} \psi_{1}\right\rangle=\left|\phi_{2} \varphi \psi\right\rangle$, with $|\varphi\rangle \neq\left|\varphi_{2}\right\rangle,|\psi\rangle \neq\left|\psi_{2}\right\rangle$, and $|\varphi \psi\rangle \neq\left|\bar{\varphi}_{2} \bar{\psi}_{2}\right\rangle$, use the same nonsingular $F^{[k]}$ as in the generic case (6) to arrive at the coefficient matrix for a general vector in $\mathfrak{W}$ :

$$
\left(\begin{array}{cccc}
\alpha \varphi_{0} \psi_{0}+\beta & \alpha \varphi_{0} \psi_{1} & \alpha \varphi_{1} \psi_{0} & \alpha \varphi_{1} \psi_{1} \\
0 & 0 & 0 & \beta
\end{array}\right)
$$

with the above conditions translated as $|\psi\rangle \neq|0\rangle \Leftrightarrow \varphi_{1} \neq 0$, $|\psi\rangle \neq|0\rangle \Leftrightarrow \psi_{1} \neq 0$, and $|\varphi \psi\rangle \neq|11\rangle \Leftrightarrow\left(\varphi_{0}, \psi_{0} \neq 0\right)$ simultaneously. It is then clear that (except for the trivial cases $\alpha \beta=0), r\left(C^{(i)}\right)=2$ for all $i=1,2,3, r\left(W_{1}\right)=2$ and $r\left(W_{2}\right)=1$. The spectrum $\sigma\left(W_{2}^{-1} W_{1}\right)$ is degenerate only if $\alpha \varphi_{0} \psi_{0}+\beta=0$. Thus there will be no $W$ vector in $\mathfrak{W}$ only when $\varphi_{0} \psi_{0}=0$-i.e., when $|\varphi\rangle=|1\rangle$ or $|\psi\rangle=|1\rangle$, which, going back before the application of the nonsingular $F^{[k]}$, means that $\left|\varphi_{1}\right\rangle=\left|\bar{\varphi}_{2}\right\rangle$ and $\left|\psi_{1}\right\rangle=\left|\bar{\psi}_{2}\right\rangle$. By using $\sigma_{x}$ upon qubits 2,3 , and 4 the symmetric case $\left|\phi_{1} \varphi_{1} \psi_{1}\right\rangle=\left|\bar{\phi}_{2} \varphi \psi\right\rangle$, with $|\varphi\rangle \neq\left|\bar{\varphi}_{2}\right\rangle$, $|\psi\rangle \neq\left|\bar{\psi}_{2}\right\rangle$, and $|\varphi \psi\rangle \neq\left|\varphi_{2} \psi_{2}\right\rangle$, is also included in this analysis. By permutations among the qubits 2, 3, and 4 all preceding cases are accounted for in a similar fashion.

(d) Finally the case $\left|\phi_{1} \varphi_{1} \psi_{1}\right\rangle=|\phi \varphi \psi\rangle$, with $|\phi\rangle \neq\left|\phi_{2}\right\rangle,\left|\bar{\phi}_{2}\right\rangle,|\varphi\rangle \neq\left|\varphi_{2}\right\rangle,\left|\bar{\varphi}_{2}\right\rangle$, and $|\psi\rangle \neq\left|\psi_{2}\right\rangle,\left|\bar{\psi}_{2}\right\rangle$, drives us, after application of the nonsingular $F^{[k]}$, to the coefficient matrix (8), already accounted for.

No more options are left; thus, we have scrutinized all possible $\mathfrak{W}$ 's with structure $\operatorname{span}\{000, \mathrm{GHZ}\}$.

\section{6. $\mathfrak{W}=\operatorname{span}\{000, W\}$}

By $\operatorname{span}\{000, W\}$ we indicate that only one 000 vector and $W$ vectors belong to $\mathfrak{W}$. The generic case will be that expressed by $\mathfrak{W}=\operatorname{span}\left\{\left|\phi_{1} \varphi_{1} \psi_{1}\right\rangle,\left|\phi_{2} \varphi_{2} \bar{\psi}_{2}\right\rangle+\left|\phi_{2} \bar{\varphi}_{2} \psi_{2}\right\rangle\right.$ $\left.+\left|\bar{\phi}_{2} \varphi_{2} \psi_{2}\right\rangle\right\}$. We will show that there will be no GHZ state in $\mathfrak{W}$ only if $\left|\phi_{1} \varphi_{1} \psi_{1}\right\rangle=\left|\phi_{2} \varphi_{2} \psi_{2}\right\rangle$, which after application of the nonsingular transformations $F^{[k]}$ will drive us to the canonical state $|0001\rangle+|0010\rangle+|0100\rangle+|1000\rangle$-i.e., the $W$ state for four qubits. As before, we will be exhaustive.

(a) If $\left|\phi_{1} \varphi_{1} \psi_{1}\right\rangle=\left|\phi_{2} \varphi_{2} \bar{\psi}_{2}\right\rangle,\left|\phi_{2} \bar{\varphi}_{2} \psi_{2}\right\rangle,\left|\bar{\phi}_{2} \varphi_{2} \psi_{2}\right\rangle$, then it is clear that a $0_{k} \Psi$ can be found in $\mathfrak{W}$ against the general hypothesis. This case is then ruled out.

(b) If $\left|\phi_{1} \varphi_{1} \psi_{1}\right\rangle=\left|\phi_{2} \varphi_{2} \psi\right\rangle$, with $|\psi\rangle \neq\left|\bar{\psi}_{2}\right\rangle$, after application of the nonsingular $F^{[k]}$, we obtain the coefficient matrix for a generic vector in $\mathfrak{W}$ :

$$
\left(\begin{array}{cccc}
\alpha \psi_{0} & \alpha \psi_{1}+\beta & \beta & 0 \\
\beta & 0 & 0 & 0
\end{array}\right)
$$

If $\alpha \psi_{1}+\beta=0$, then there will exist non-null $\alpha, \beta$ such that a $0_{3} \Psi$ vector will belong to $\mathfrak{W}$, against the hypothesis. Thus it must be $\psi_{1}=0$-i.e., $|\psi\rangle=|0\rangle$. Under this restriction and after using theorem 1 upon the coefficient matrix (10), all vectors will be of type $W$, since $r\left(C^{(i)}\right)=2$ for all $i=1,2,3$, $r\left(W_{1}\right)=2, r\left(W_{2}\right)=1$, and the spectrum $\sigma\left(W_{1}^{-1} W_{2}\right)$ is always degenerate. By permutation symmetry the cases $\left|\phi_{1} \varphi_{1} \psi_{1}\right\rangle$ $=\left|\phi_{2} \varphi \psi_{2}\right\rangle,\left|\phi \varphi_{2} \psi_{2}\right\rangle$, with the corresponding restrictions, are also considered in this analysis.

(c) If $\left|\phi_{1} \varphi_{1} \psi_{1}\right\rangle=\left|\phi_{2} \varphi \bar{\psi}_{2}\right\rangle$, a similar argument leads to the coefficient matrix for a generic vector in $\mathfrak{W}$ given by

$$
\left(\begin{array}{cccc}
0 & \alpha \varphi_{0}+\beta & \beta & 0 \alpha \varphi_{1} \\
\beta & 0 & 0 & 0
\end{array}\right)
$$

After application of theorem 1, it should be clear that it is always possible to find non-null $\alpha, \beta$ such that (11) corresponds to a GHZ vector in $\mathfrak{W}$, against the hypothesis. By permutation symmetry, the rest of cases with the 000 generator having two factors in common with some of components of the $W$ generator is also contained in this analysis.

(d) If $\left|\phi_{1} \varphi_{1} \psi_{1}\right\rangle=\left|\phi_{1} \varphi \psi\right\rangle$, with $|\varphi\rangle \neq\left|\varphi_{2}, \bar{\varphi}_{2}\right\rangle$ and $|\psi\rangle \neq\left|\psi_{2}, \bar{\psi}_{2}\right\rangle$, after a similar argument the coefficient matrix of a generic vector in the right singular subspace will be

$$
\left(\begin{array}{cccc}
\alpha \varphi_{0} \psi_{0} & \alpha \varphi_{0} \psi_{1}+\beta & \alpha \varphi_{1} \psi_{0}+\beta & \alpha \varphi_{1} \psi_{1} \\
0 & 0 & 0 & \beta
\end{array}\right) .
$$

It should be clear that $r\left(C^{(i)}\right)=2$ for all $i=1,2,3$. Now, if $\varphi_{1} \psi_{0}+\varphi_{0} \psi_{1} \neq 0$, there will always exist non-null $\alpha, \beta$ such that $r\left(W_{1}\right)=r\left(W_{2}\right)=1$, hence a GHZ vector, against the hypothesis. On the contrary, if $\varphi_{1} \psi_{0}+\varphi_{0} \psi_{1}=0$, then $r\left(W_{1}\right)=2$ and $r\left(W_{2}\right)=1$, but it is always possible to find non-null $\alpha, \beta$ such that $\sigma\left(W_{1}^{-1} W_{2}\right)$ is nondegenerate; hence, GHZ $\in \mathfrak{W}$. Notice that at most another $W$ vector, apart from the generator, can be found. By permutation symmetry and by using $\sigma_{x}$ upon each qubit, the rest of cases in which the 000 generator contains one common factor vector with the $W$ generator is also considered in this analysis.

(e) Finally, if $\left|\phi_{1} \varphi_{1} \psi_{1}\right\rangle=|\phi \varphi \psi\rangle$, with no common factor vector with the $W$ generator, a similar argument leads to the coefficient matrix for a generic vector in the right singular subspace given by

$$
\left(\begin{array}{cccc}
\alpha \phi_{0} \varphi_{0} \psi_{0} & \alpha \phi_{0} \varphi_{0} \psi_{1}+\beta & \alpha \phi_{0} \varphi_{1} \psi_{0}+\beta & \alpha \phi_{0} \varphi_{1} \psi_{1} \\
\alpha \phi_{1} \varphi_{0} \psi_{0}+\beta & \alpha \phi_{1} \varphi_{0} \psi_{1} & \alpha \phi_{1} \varphi_{1} \psi_{0} & \alpha \phi_{1} \varphi_{1} \psi_{1}
\end{array}\right) .
$$

A systematic application of theorem 1 makes it clear that there always exist $\alpha, \beta$ such that this matrix corresponds to a GHZ state, against the hypothesis.

\section{7. $\mathfrak{W}=\operatorname{span}\left\{0_{1} \Psi_{23}, 0_{1} \Psi_{23}\right\}$}

The generic case will be $\mathfrak{W}=\operatorname{span}\left\{\left|\phi_{1} \Psi_{1}\right\rangle,\left|\phi_{2} \Psi_{2}\right\rangle\right\}$, where $\left|\Psi_{k}\right\rangle$ denotes a bipartite entangled vector. Several cases appear.

(i) When $\left|\phi_{1}\right\rangle$ and $\left|\phi_{2}\right\rangle$ are linearly dependent, then $\mathfrak{W}=|\phi\rangle \otimes \operatorname{span}\left\{\left|\Psi_{1}\right\rangle,\left|\Psi_{2}\right\rangle\right\}$, driving us to the already known degenerate classes $0_{1} 0_{2} \Psi, 0_{2} \mathrm{GHZ}$ and $0_{2} W$, respectively, 
when it adopts the structures $\operatorname{span}\{|\phi \Psi\rangle\}, \quad|\phi\rangle$ $\otimes \operatorname{span}\{00,00\}$, and $|\phi\rangle \otimes \operatorname{span}\{00, \Psi\}$. In the forthcoming cases, we will then impose the linear independence of $\left|\phi_{1}\right\rangle$ and $\left|\phi_{2}\right\rangle$.

(ii) If $\left|\Psi_{1}\right\rangle$ and $\left|\Psi_{2}\right\rangle$ are linearly dependent, then $\mathfrak{W}=\mathbb{C}^{2}$ $\otimes|\Psi\rangle$, corresponding to the degenerate class $\Psi_{12} \Psi_{34}$, as stated above. Therefore, we will also impose the linear independence of $\left|\Psi_{1}\right\rangle$ and $\left|\Psi_{2}\right\rangle$.

(iii) When $\operatorname{span}\left\{\left|\Psi_{1}\right\rangle,\left|\Psi_{2}\right\rangle\right\}=\operatorname{span}\{00,00\}$, then it is always possible to find linear independent factor vectors such that $\left|\Psi_{j}\right\rangle=a_{j 1}|\varphi \psi\rangle+a_{j 2}|\bar{\varphi} \bar{\psi}\rangle$, with $a_{j 1} a_{j 2} \neq 0$. Defining nonsingular $F^{[k]}$ such that $F^{[2]}\left[a_{11}\left|\phi_{1}\right\rangle\right]=|0\rangle$, $F^{[2]}\left[a_{21}\left|\phi_{2}\right\rangle\right]=|1\rangle, \quad F^{[3]}[|\varphi\rangle]=|0\rangle, \quad F^{[3]}[|\bar{\varphi}\rangle]=|1\rangle, \quad F^{[4]}[|\psi\rangle]$ $=|0\rangle, F^{[4]}[|\bar{\psi}\rangle]=|1\rangle$, and $F^{[1]}$ as in preceding cases, we obtain the canonical matrix

$$
\left(\begin{array}{cccccccc}
1 & 0 & 0 & \lambda_{1} & 0 & 0 & 0 & 0 \\
0 & 0 & 0 & 0 & 1 & 0 & 0 & \lambda_{2}
\end{array}\right),
$$

where we have defined $\lambda_{i} \equiv \frac{a_{i 2}^{*}}{a_{i 1}^{*}}$. This matrix corresponds to the canonical state $|0000\rangle+|1100\rangle+\lambda_{1}|0011\rangle+\lambda_{2}|1111\rangle$, with $\lambda_{1} \neq \lambda_{2}$.

(iv) When $\operatorname{span}\left\{\left|\Psi_{1}\right\rangle,\left|\Psi_{2}\right\rangle\right\}=\operatorname{span}\{00, \Psi\}$, then it is always possible to find linear independent factor vectors such that $\left|\Psi_{j}\right\rangle=a_{j 1}|\varphi \psi\rangle+a_{j 2}(|\varphi \bar{\psi}\rangle+|\bar{\varphi} \psi\rangle)$, with $a_{j 1} a_{j 2} \neq 0$. Defining nonsingular $F^{[k]}$ such that $F^{[2]}\left[a_{11}\left|\phi_{1}\right\rangle\right]=|0\rangle$, $F^{[2]}\left[a_{21}\left|\phi_{2}\right\rangle\right]=|1\rangle, \quad F^{[3]}[|\varphi\rangle]=|0\rangle, \quad F^{[3]}[|\bar{\varphi}\rangle]=|1\rangle, \quad F^{[4]}[|\psi\rangle]$ $=|0\rangle, F^{[4]}[|\bar{\psi}\rangle]=|1\rangle$, and $F^{[1]}$ as in preceding cases, we obtain the canonical matrix

$$
\left(\begin{array}{cccccccc}
1 & \lambda_{1} & \lambda_{1} & 0 & 0 & 0 & 0 & 0 \\
0 & 0 & 0 & 0 & 1 & \lambda_{2} & \lambda_{2} & 0
\end{array}\right),
$$

where we have defined $\lambda_{i} \equiv \frac{a_{i 2}^{*}}{a_{i 1}^{*}}$. This matrix corresponds to the canonical state $|0000\rangle+|1100\rangle+\lambda_{1}|0001\rangle+\lambda_{1}|0010\rangle$ $+\lambda_{2}|1101\rangle+\lambda_{2}|1110\rangle$, with $\lambda_{1} \neq \lambda_{2}$.

All possible cases have been considered. Furthermore, notice that several states obtained by permutations involving qubit 1 in preceding cases are enclosed in this class. This will also happen in forthcoming classes: some permutations involving qubit 1 usually involve a change of entanglement class, although the general structure of the canonical state shows permutation symmetry [12].

\section{8. $\mathfrak{W}=\operatorname{span}\left\{0_{1} \Psi_{23}, 0_{2} \Psi_{13}\right\}$}

The generic case is given by $\mathfrak{W}=\operatorname{span}\left\{\left|\phi_{1} \Psi_{1}\right\rangle,\left|\phi_{2} \varphi_{2} \psi_{2}\right\rangle\right.$ $\left.+\left|\bar{\phi}_{2} \varphi_{2} \bar{\psi}_{2}\right\rangle\right\}$. Two possibilities arise.

(i) $\operatorname{span}\left\{\left|\Psi_{1}\right\rangle,\left|\varphi_{2} \psi_{2}\right\rangle\right\}=\operatorname{span}\{00,00\}=\operatorname{span}\left\{\left|\varphi_{2} \psi_{2}\right\rangle\right.$, $\left.\left|\bar{\varphi}_{2} \overline{\bar{\psi}}_{2}\right\rangle\right\}$, where the double overbar also indicates linear independence with respect to $\left|\psi_{2}\right\rangle$, although possible linear dependence with $\left|\bar{\psi}_{2}\right\rangle$. Then a generic vector in $\mathfrak{W}$ will be given by $\alpha\left|\phi_{1}\right\rangle\left(a\left|\varphi_{2} \psi_{2}\right\rangle+b\left|\bar{\varphi}_{2} \overline{\bar{\psi}}_{2}\right\rangle\right)+\beta\left(\left|\phi_{2} \varphi_{2} \psi_{2}\right\rangle\right.$ $\left.+\left|\bar{\phi}_{2} \varphi_{2} \bar{\psi}_{2}\right\rangle\right)$,with

$a b \neq 0$. Defining nonsingular $F^{[k]}$ such that $F^{[2]}\left[\left|\phi_{2}\right\rangle\right]=|0\rangle$, $F^{[2]}\left[\left|\bar{\phi}_{2}\right\rangle\right]=|1\rangle, \quad F^{[3]}\left[\left|\varphi_{2}\right\rangle\right]=|0\rangle, \quad F^{[3]}\left[\frac{b}{a}\left|\bar{\varphi}_{2}\right\rangle\right]=|1\rangle, \quad F^{[4]}\left[\left|\psi_{2}\right\rangle\right]$ $=|0\rangle, F^{[4]}\left[\left|\bar{\psi}_{2}\right\rangle\right]=|1\rangle$, and $F^{[1]}$ as in preceding cases, we obtain the canonical vector $|0 \phi 00\rangle+|0 \phi 1 \psi\rangle+|1000\rangle+|1101\rangle$, with the restrictions that $|\psi\rangle \neq 0$ and $|\phi\rangle \neq|0,1\rangle$, simultaneously (otherwise we would be reconsidering previous classes). With the use of $\sigma_{x}$ upon qubit 4 , we are also embracing the case in which $\operatorname{span}\left\{\left|\Psi_{1}\right\rangle,\left|\varphi_{2} \bar{\psi}_{2}\right\rangle\right\}=\operatorname{span}\{00,00\}$ $=\operatorname{span}\left\{\left|\varphi_{2} \bar{\psi}_{2}\right\rangle,\left|\bar{\varphi}_{2} \overline{\bar{\psi}}_{2}\right\rangle\right\}$, where now the double overbar denotes linear independence with respect to $\left|\bar{\psi}_{2}\right\rangle$.

(ii) $\operatorname{span}\left\{\left|\Psi_{1}\right\rangle,\left|\varphi_{2} \psi_{2}\right\rangle\right\}=\operatorname{span}\{00, \Psi\}=\operatorname{span}\left\{\left|\varphi_{2} \psi_{2}\right\rangle,\left|\varphi_{2} \overline{\bar{\psi}}_{2}\right\rangle\right.$ $\left.+\left|\bar{\varphi}_{2} \psi_{2}\right\rangle\right\}$, with the same convention as before for the double overbar. Expressing $\left|\Psi_{1}\right\rangle=a\left|\varphi_{2} \psi_{2}\right\rangle+b\left(\left|\varphi_{2} \overline{\bar{\psi}}_{2}\right\rangle+\left|\bar{\varphi}_{2} \psi_{2}\right\rangle\right)$ and with a similar argument as the preceding case, we arrive at the canonical state $|0 \phi 0 \psi\rangle+|0 \phi 10\rangle+|1000\rangle+|1101\rangle$, with the restrictions that $|\psi\rangle \neq 0$ and $|\phi\rangle \neq|0,1\rangle$, simultaneously (otherwise we would be reconsidering previous classes). By using $\sigma_{x}$ upon qubit 4 , we are also considering the case $\operatorname{span}\left\{\left|\Psi_{1}\right\rangle,\left|\psi_{2} \bar{\phi}_{2}\right\rangle\right\}=\operatorname{span}\{00, \Psi\}$.

\section{9. $\mathfrak{W}=\operatorname{span}\left\{0_{1} \Psi_{23}, 0_{3} \Psi_{12}\right\}$}

Although this case can also be analyzed with similar arguments, we will use permutation symmetry among qubits 2 and 3 in the case $\operatorname{span}\left\{0_{1} \Psi_{23}, 0_{2} \Psi_{13}\right\}$ to arrive at the canonical states $|0 \phi 00\rangle+|0 \phi \varphi 1\rangle+|1000\rangle+|1110\rangle$ and $|0 \phi \varphi 0\rangle$ $+|0 \phi 01\rangle+|1000\rangle+|1110\rangle$.

\section{0. $\mathfrak{W}=\operatorname{span}\left\{0_{2} \Psi_{13}, 0_{2} \Psi_{13}\right\}$}

Under permutation symmetry between qubits 2 and 3 in the case $\operatorname{span}\left\{0_{1} \Psi_{23}, 01 \Psi_{23}\right\}$, we rapidly find two genuinely entangled canonical states-namely, $\quad|0000\rangle+|1010\rangle$ $+\lambda_{1}|0101\rangle+\lambda_{2}|1111\rangle$ and $|0000\rangle+|1010\rangle+\lambda_{1}|0101\rangle$ $+\lambda_{1}|0001\rangle+\lambda_{2}|1011\rangle+\lambda_{2}|1110\rangle$-and one degenerate canonical state of type $\Psi_{13} \Psi_{24}$ (apart from the already considered).

\section{1. $\mathfrak{W}=\operatorname{span}\left\{0_{2} \Psi_{13}, 0_{3} \Psi_{12}\right\}$}

Under permutation symmetry between qubits 2 and 4 in the case $\operatorname{span}\left\{0_{1} \Psi_{23}, 0_{3} \Psi_{12}\right\}$ we rapidly find two genuinely entangled canonical states: namely, $|000 \psi\rangle+|0 \phi 1 \psi\rangle+|1000\rangle$ $+|1101\rangle$ and $|0 \phi 0 \psi\rangle+|001 \psi\rangle+|1000\rangle+|1101\rangle$.

\section{2. $\mathfrak{W}=\operatorname{span}\left\{0_{3} \Psi_{12}, 0_{3} \Psi_{12}\right\}$}

Under permutation symmetry between qubits 2 and 4 in the case $\operatorname{span}\left\{0_{1} \Psi_{23}, 0_{1} \Psi_{23}\right\}$, we rapidly find two genuinely entangled canonical states-namely, $|0000\rangle+|1001\rangle$ $+\lambda_{1}|0110\rangle+\lambda_{2}|1111\rangle$ and $|0000\rangle+|1001\rangle+\lambda_{1}|0010\rangle$ $+\lambda_{1}|0100\rangle+\lambda_{2}|1011\rangle+\lambda_{2}|1101\rangle$-and one degenerate canonical state of type $\Psi_{14} \Psi_{23}$ (apart from that already considered).

\section{3. $\mathfrak{W}=\operatorname{span}\left\{0_{1} \Psi_{23}, G H Z\right\}$}

The generic case will be of the form $\mathfrak{W}$ $=\operatorname{span}\left\{\left|\phi_{1} \Psi_{1}\right\rangle,\left|\phi_{2} \varphi_{2} \psi_{2}\right\rangle+\left|\bar{\phi}_{2} \bar{\varphi}_{2} \bar{\psi}_{2}\right\rangle\right\}$. Finding nonsingular transformations $F^{[i]}$ as before, we can write $\mathfrak{W}$ $=\operatorname{span}\{|\phi \Psi\rangle,|000\rangle+|111\rangle\}$. A generic vector in $\mathfrak{W}$ will then 
TABLE I. Genuine entanglement classes for four qubits.

\begin{tabular}{lccc}
\hline \hline Class $(\mathfrak{W})$ & Canonical states & Name & Proposed notation \\
\hline $\operatorname{span}\{000,000\}$ & $|0000\rangle+|1111\rangle$ & $\mathrm{GHZ}$ & $\mathfrak{W}_{000,000}$ \\
$\operatorname{span}\left\{000,0_{k} \Psi\right\}$ & $|0000\rangle+|1100\rangle+|1111\rangle$ & $\mathfrak{W}_{000,0_{k} \Psi}$ \\
$\operatorname{span}\{000, \mathrm{GHZ}\}$ & $|0000\rangle+|1101\rangle+|1110\rangle$ & \\
$\operatorname{span}\{000, W\}$ & $|0 \phi \varphi \psi\rangle+|1000\rangle+|1111\rangle$ & $\mathfrak{W}_{000, \mathrm{GHZ}}$ \\
$\operatorname{span}\left\{0_{k} \Psi, 0_{k} \Psi\right\}$ & $|0000\rangle+|1100\rangle+\lambda_{1}|0011\rangle+\lambda_{2}|1111\rangle$ & $\mathfrak{W}_{000, W}$ \\
& $|0000\rangle+|1100\rangle+\lambda_{1}|0001\rangle+\lambda_{1}|0010\rangle+\lambda_{2}|1101\rangle$ & & $\Phi_{4}$ \\
$\operatorname{span}\left\{0_{i} \Psi, 0_{j} \Psi\right\}$ & $|0 \phi 00\rangle+|0 \phi 1 \psi\rangle+|1000\rangle+|1101\rangle$ & & \\
$\operatorname{span}\left\{0_{k} \Psi, \mathrm{GHZ}\right\}$ & $|0 \phi 0 \psi\rangle+|0 \phi 10\rangle+|1000\rangle+|1101\rangle$ & & $\mathfrak{W}_{0_{k}} \Psi, 0_{k} \Psi$ \\
$\operatorname{span}\{\mathrm{GHZ}, W\}$ & $|0 \phi \Psi\rangle+|1000\rangle+|1111\rangle$ & & \\
\hline \hline
\end{tabular}

be of the form $\alpha|\phi \Psi\rangle+\beta(|000\rangle+|111\rangle)$. The restrictions on $|\phi\rangle$ and $|\Psi\rangle$ (thus on $\left|\phi_{1}\right\rangle$ and $\left|\Psi_{1}\right\rangle$ ) will be that no other $0_{i_{k}} \Psi$ must belong to $\mathfrak{W}$. For instance, a particular restriction will be that $|\Psi\rangle \notin \operatorname{span}\{00,11\}$, since otherwise $|\Psi\rangle=a|00\rangle$ $+b|11\rangle, a b \neq 0$, and then a generic state in $\mathfrak{W}$ will adopt the form $(\alpha a|\phi\rangle+\beta|0\rangle)|00\rangle+(\alpha b|\phi\rangle+\beta|1\rangle)|11\rangle$. It is always possible to find non-null $\alpha, \beta$ such that the preceding state is a $0_{1} \Psi_{23}$ vector different to $|\phi \Psi\rangle$-i.e., another $0_{1} \Psi_{23}$ vector: we would be in the $\operatorname{span}\left\{0_{1} \Psi_{23}, 0_{1} \Psi_{23}\right\}$ case. The canonical state will be given by $|0 \phi \Psi\rangle+|1000\rangle+|1111\rangle$.

\section{4. $\mathfrak{W}=\operatorname{span}\left\{0_{2} \Psi_{13}, G H Z\right\}$}

By permutation symmetry between qubits 2 and 4, the canonical state will be $\left|0 \Psi_{24} \phi_{3}\right\rangle+|1000\rangle+|1111\rangle$, where the first factor denotes an entangled state between qubits 2 and 4 and two factor states involving state 1 and 3 .

\section{5. $\mathfrak{W}=\operatorname{span}\left\{0_{3} \Psi_{12}, G H Z\right\}$}

By permutation symmetry between qubits 2 and 3, the canonical state will be $\left|0 \Psi_{23} \phi_{4}\right\rangle+|1000\rangle+|1111\rangle$, where the first factor denotes an entangled state between qubits 2 and 3 and two factor states involving state 1 and 4 .

\section{6. $\mathfrak{W}=\operatorname{span}\left\{0_{1} \Psi_{23}, W\right\}$}

By $\mathfrak{W}=\operatorname{span}\left\{0_{1} \Psi_{23}, W\right\}$ it is meant that only one degenerate tripartite vector of type $0_{1} \Psi_{23}$ belongs to $\mathfrak{W}$, all the rest being of type $W$. It is immediate to prove that this is impossible-i.e., that there is always possible to find a $\mathrm{GHZ}$ vector in $\mathfrak{W}$. The generic case will be given by $\mathfrak{W}$ $=\operatorname{span}\left\{\left|\phi_{1} \varphi_{1} \psi_{1}\right\rangle,\left|\phi_{2} \varphi_{2} \bar{\psi}_{2}\right\rangle+\left|\phi_{2} \bar{\varphi}_{2} \psi_{2}\right\rangle+\left|\bar{\phi}_{2} \varphi_{2} \psi_{2}\right\rangle\right\}$. Defining $F^{[k]}$ so that $F^{[2]}\left|\psi_{2}\right\rangle=|0\rangle, F^{[2]}\left|\bar{\phi}_{2}\right\rangle=|1\rangle$ and similarly for $k$ $=3,4$ and using the same $F^{[1]}$ as in preceding cases, the coefficient matrix for a generic [13] vector $|\phi \Psi\rangle+\lambda(|000\rangle$ $+|111\rangle)$ in $\mathfrak{W}$ will be

$$
\left(\begin{array}{cccc}
\phi_{0} \Psi_{00} & \phi_{0} \Psi_{01}+\lambda & \phi_{0} \Psi_{10}+\lambda & \phi_{0} \Psi_{11} \\
\phi_{1} \Psi_{00}+\lambda & \phi_{1} \Psi_{01} & \phi_{1} \Psi_{10} & \phi_{1} \Psi_{11}
\end{array}\right) .
$$

Now there is a continuous range of values $\lambda \in \mathbb{C}$ such that $r\left(C^{(i)}\right)=2$. Focus upon the set of which such that $\operatorname{det} W_{2} \neq 0$ (again a continuous range of them); then, it is clear that the spectrum of $W_{2}^{-1} W_{1}$ will be in general nondegenerate, driving us to a GHZ vector. Thus we are in fact in the case $\operatorname{span}\left\{0_{1} \Psi_{23}, \mathrm{GHZ}\right\}$.

\section{7. $\mathfrak{W}=\operatorname{span}\left\{0_{2} \Psi_{13}, W\right\}$}

By permutation symmetry the same analysis as in the preceding case allows us to conclude that we are in the $\operatorname{span}\left\{0_{2} \Psi_{13}, \mathrm{GHZ}\right\}$ case.

\section{8. $\mathfrak{W}=\operatorname{span}\left\{0_{3} \Psi_{12}, W\right\}$}

By permutation symmetry the same analysis as in the preceding case allows us to conclude that we are in the $\operatorname{span}\left\{0_{3} \Psi_{12}, \mathrm{GHZ}\right\}$ case.

\section{9. $\mathfrak{W}=\operatorname{span}\{G H Z, W\}$}

In this class there exist no degenerate tripartite vectors; i.e., all of them are either of type GHZ or of type $W$. With the same arguments as before, the canonical state is found to be of the form $|1 \phi \varphi \psi\rangle+|1 \bar{\phi} \bar{\varphi} \bar{\psi}\rangle+|0001\rangle+|0010\rangle+|0100\rangle$, where the product vectors $|\phi\rangle,|\bar{\phi}\rangle,|\varphi\rangle,|\bar{\varphi}\rangle,|\psi\rangle$, and $|\bar{\psi}\rangle$ are restricted to produce no degenerate state in $\mathfrak{W}$.

\section{0. $\mathfrak{W}=\operatorname{span}\{G H Z, G H Z\}$}

The notation is meant to describe the situation in which only GHZ vectors are contained in $\mathfrak{W}$. Apart for particular instances in which a degenerate tripartite vector may appear as a linear combination of both GHZ generators (which we rule out, since they have been already dealt with), we will show that there will always exist at least one $W$ vector in $\mathfrak{W}$. The generic case is given by $\operatorname{span}\left\{\left|\phi_{1} \varphi_{1} \psi_{1}\right\rangle\right.$ $\left.+\left|\bar{\phi}_{1} \bar{\varphi}_{1} \bar{\psi}_{1}\right\rangle,\left|\phi_{2} \varphi_{2} \psi_{2}\right\rangle+\left|\bar{\phi}_{2} \bar{\varphi}_{2} \bar{\psi}_{2}\right\rangle\right\}$. Then, after defining nonsingular transformations $F^{[k]}$ in a similar fashion as in all preceding cases, the coefficient matrix for a generic vector in $\mathfrak{W}$ will be given by 


$$
\left(\begin{array}{cccc}
\phi_{0} \varphi_{0} \psi_{0}+\bar{\phi}_{0} \bar{\varphi}_{0} \bar{\psi}_{0}+\lambda & \phi_{0} \varphi_{0} \psi_{1}+\bar{\phi}_{0} \bar{\varphi}_{0} \bar{\psi}_{1} & \phi_{0} \varphi_{1} \psi_{0}+\bar{\phi}_{0} \bar{\varphi}_{1} \bar{\psi}_{0} & \phi_{0} \varphi_{1} \psi_{1}+\bar{\phi}_{0} \bar{\varphi}_{1} \bar{\psi}_{1} \\
\phi_{1} \varphi_{0} \psi_{0}+\bar{\phi}_{1} \bar{\varphi}_{0} \bar{\psi}_{0} & \phi_{1} \varphi_{0} \psi_{1}+\bar{\phi}_{1} \bar{\varphi}_{0} \bar{\psi}_{1} & \phi_{1} \varphi_{1} \psi_{0}+\bar{\phi}_{1} \bar{\varphi}_{1} \bar{\psi}_{0} & \phi_{1} \varphi_{1} \psi_{1}+\bar{\phi}_{1} \bar{\varphi}_{1} \bar{\psi}_{1}+\lambda
\end{array}\right)
$$

There is a continuous range of values $\lambda$ such that $r\left(C^{(i)}\right)$ $=2$ for all $i=1,2,3$. Focus upon the set of which such that $r\left(W_{1}\right)=2$; then, there will always exist a value $\lambda^{*}$ such that $\sigma\left(W_{1}^{-1} W_{2}\right)$ is degenerate $\left[\lambda^{*}\right.$ is the solution to the equation $\Delta\left(W_{1}^{-1} W_{2}\right)=0$, where $\Delta(A)$ denotes the discriminant of the characteristic polynomial of the matrix $A]$. We are then always in the case $\operatorname{span}\{\mathrm{GHZ}, W\}$.

\section{1. $\mathfrak{W}=\operatorname{span}\{W, W\}$}

The notation is meant to describe the situation in which only $W$ vectors are contained in $\mathfrak{W}$. Apart for particular in- stances in which a degenerate tripartite vector may appear as a linear combination of both $W$ generators (which we rule out, since they have been already dealt with), we will show that there will always exist at least one GHZ vector in $\mathfrak{W}$. The generic case is given by $\operatorname{span}\left\{\left|\phi_{1} \varphi_{1} \bar{\psi}_{1}\right\rangle\right.$ $\left.+\left|\phi_{1} \bar{\varphi}_{1} \psi_{1}\right\rangle,\left|\bar{\phi}_{1} \varphi_{1} \psi_{1}\right\rangle,\left|\phi_{2} \varphi_{2} \bar{\psi}_{2}\right\rangle+\left|\phi_{2} \bar{\varphi}_{2} \psi_{2}\right\rangle,\left|\bar{\phi}_{2} \varphi_{2} \psi_{2}\right\rangle\right\}$. Then, after defining nonsingular transformations $F^{[k]}$ in a similar fashion as in all preceding cases, the coefficient matrix for a genericvector in $\mathfrak{W}$ will be given by $\left(\begin{array}{cccc}\phi_{0} \varphi_{0} \bar{\psi}_{0}+\phi_{0} \bar{\varphi}_{0} \psi_{0}+\bar{\phi}_{0} \varphi_{0} \psi_{0} & \phi_{0} \varphi_{0} \bar{\psi}_{1}+\phi_{0} \bar{\varphi}_{0} \psi_{1}+\bar{\phi}_{0} \varphi_{0} \psi_{1}+\lambda & \phi_{0} \varphi_{1} \bar{\psi}_{0}+\phi_{0} \bar{\varphi}_{1} \psi_{0}+\bar{\phi}_{0} \varphi_{1} \psi_{0}+\lambda & \phi_{0} \varphi_{1} \bar{\psi}_{1}+\phi_{0} \bar{\varphi}_{1} \psi_{1}+\bar{\phi}_{0} \varphi_{1} \psi_{1} \\ \phi_{1} \varphi_{0} \bar{\psi}_{0}+\phi_{1} \bar{\varphi}_{0} \psi_{0}+\bar{\phi}_{1} \varphi_{0} \psi_{0}+\lambda & \phi_{1} \varphi_{0} \bar{\psi}_{1}+\phi_{1} \bar{\varphi}_{0} \psi_{1}+\bar{\phi}_{1} \varphi_{0} \psi_{1} & \phi_{1} \varphi_{1} \bar{\psi}_{0}+\phi_{1} \bar{\varphi}_{1} \psi_{0}+\bar{\phi}_{1} \varphi_{1} \psi_{0} & \phi_{1} \varphi_{1} \bar{\psi}_{1}+\phi_{1} \bar{\varphi}_{1} \psi_{1}+\bar{\phi}_{1} \varphi_{1} \psi_{1}\end{array}\right)$.

There is a continuous range of vales $\lambda$ such that $r\left(C^{(i)}\right)$ $=2$ for all $i=1,2,3$. Focus upon the set of which such that $r\left(W_{2}\right)=2$; then, there will always exist values $\lambda^{*}$ such that $\sigma\left(W_{1}^{-1} W_{2}\right)$ is nondegenerate $\left[\lambda^{*}\right.$ is not the solution to the equation $\Delta\left(W_{2}^{-1} W_{1}\right)=0$, where $\Delta(A)$ denotes the discriminant of the characteristic polynomial of the matrix $A]$. We are then always in the case $\operatorname{span}\{\mathrm{GHZ}, W\}$, too.

\section{Counting entanglement classes}

The preceding classification of states under the SLOCC criterion reveals a block structure for the entire state space $\left(C^{2}\right)^{\otimes 4}$, with a continuous range of nonequivalent vectors inside each block (except for some of them). Considering each block as an entanglement class itself, then we have found 18 degenerate and 16 genuine classes-i.e., a total of 34 entanglement classes.

Furthermore, taking into account permutation symmetry among the four qubits, indeed we have proven that four qubits can be entangled in eight inequivalent ways. This is in apparent contradiction to the result found in [8], where four qubits are claimed to be entangled in nine possible ways. A closer look at this work reveals that one of the blocks in that classification contains no genuinely entangled vector: namely the class $L_{0_{3 \oplus 1} 0_{3 \oplus 1}}$ with canonical state $|0000\rangle$ $+|0111\rangle$. We would like to express our conviction that this difference stems from a distinct way of counting or considering entanglement classes. We believe ours shows a greater agreement with the philosophy of the seminal paper by Dür et al. [6]. Thus we have proven the following.

Theorem 2. Let $|\Phi\rangle$ be a genuinely entangled four-partite pure state. Then $|\Phi\rangle$ belongs to one of the following eight genuine entanglement classes and is equivalent to some of its canonical vectors, according to Table I:

The canonical vectors obtained by permutation among qubits 2, 3, and 4 have been omitted in each entry. Restrictions upon the range of the free parameters in each class amounts to not producing a precedent structure for the right singular subspace $\mathfrak{W}$.

Names should be given to all classes. A systematic notation for these classes could possibly be $\mathfrak{W}_{\Psi_{i}, \Psi_{j}}$, where the subindices indicate the generators of $\mathfrak{W}$. In this convention $\mathrm{GHZ}=\mathfrak{W}_{000,000}, W=\mathfrak{W}_{000, W}$, and $\Phi_{4}=\mathfrak{W}_{0_{1} \Psi, 0_{1} \Psi}$. However, a further discrimination referring to the particular canonical state must be explicitly stated.

\section{CONCLUDING REMARKS}

We have given the classification of entangled states of four qubits under stochastic local operations and classical communication using the inductive approach introduced in [7]. This approach is based on the analysis of the structure of the right singular subspace $\mathfrak{W}$ of the coefficient matrix of the state in a product basis according to the entanglement classes for three qubits.

Agreeing to consider each structure of $\mathfrak{W}$ as a single entanglement class, we have found 18 degenerate and 16 genuine classes (total of 34 classes), where permutation is explicitly included in the counting. Taking into account the permutation among the qubits, eight genuine classes can be considered, compiled in Table I. As expected, in most of the 
classes a continuous range of strictly nonequivalent states is contained, although with similar structure.

This result allows us to predict that there will be at most 765 entanglement classes (permutation included) for 5-partite systems: 595 at most genuine and 170 at most degenerate (cf. [7]). No doubt, this classification stands up as a formidable task that our inductive method may help to elucidate.

\section{ACKNOWLEDGMENTS}

L.L. and J.L. acknowledge financial support from the Spanish MEC projects No. FIS2005-05304, D.S. from No. FIS2004-01576, and E.S. from EU RESQ, EuroSQIP, and DFG SFB631 projects. L.L. also acknowledges support from FPU Grant No. AP2003-0014.
[1] J. Bell, Speakable and Unspeakable in Quantum Mechanics (Cambridge University Press, Cambridge, England, 1987).

[2] A. Peres, Quantum Theory: Concepts and Methods (Kluwer, Dordrecht, 1993).

[3] The Physics of Quantum Information, edited by D. Bouwmeester, A. Ekert, and A. Zeilinger (Springer, Berlin, 2000).

[4] M. Nielsen and I. Chuang, Quantum Computation and Quantum Information (Cambridge University Press, Cambridge, England, 2000).

[5] Quantum Information: An Introduction to Basic Theoretical Concepts and Experiments, edited by G. Alber, T. Beth, M. Horodecki, P. Horodecki, R. Horodecki, M. Rötteler, H. Weinfurter, R. Werner, and A. Zeilinger (Springer, Berlin, 2001), Chap. 5.

[6] W. Dür, G. Vidal, and J. I. Cirac, Phys. Rev. A 62, 062314 (2000)
[7] L. Lamata, J. León, D. Salgado, and E. Solano, Phys. Rev. A 74, 052336 (2006).

[8] F. Verstraete, J. Dehaene, B. De Moor, and H. Verschelde, Phys. Rev. A 65, 052112 (2002).

[9] M. F. Cornelio and A. F. R. de Toledo Piza, Phys. Rev. A 73, 032314 (2006).

[10] A. Sanpera, R. Tarrach, and G. Vidal, Phys. Rev. A 58, 826 (1998).

[11] Irrelevant complex conjugation upon each factor has been omitted for ease of notation.

[12] This already happened in the tripartite classification: classes $0_{i_{k}} \Psi$ drive us to different right singular subspaces, despite the permutation symmetry between the three qubits. The ultimate reason is the singularization of qubit 1 produced by the choice of the representation of the coefficient matrix $C^{(1)}$.

[13] Up to the $0_{1 \Psi}$ generator. 